\title{
Modelado y simulación de la deformación plástica del aluminio ASTM a1200 durante el proceso de conformado mecánico
}

\section{(Modeling and simulation of plastic deformation of Aluminium ASTM A1200 during mechanical conformed process)}

\author{
Luis C. Juiña, ${ }^{1}$ Víctor H. Cabrera, ${ }^{1}$ Nancy V. Moreno, ${ }^{1}$ Christian A. Anrango ${ }^{1}$
}

\begin{abstract}
Resumen
La investigación tiene por finalidad evaluar el comportamiento de un material al ser sometido a una deformación plástica. El proceso estuvo compuesto de una fase analítica realizada mediante formulaciones previas y por un proceso de integración con el fin de determinar el desarrollo en plano de una pieza conformada, para ello se establecieron los esfuerzos generados y cambios de sección durante el proceso de embutido, se utilizó software de simulación basado en elementos finitos para evaluar el comportamiento del material en el proceso mencionado. En la parte experimental se determinó las características mecánicas del material, aluminio ASTM A1200, mediante el ensayo normalizado, el límite de fluencia fue de $214 \mathrm{MPa}$. El proceso de conformado se efectúo en una prensa de 5 t con dispositivo neumático, se realizaron varios ensayos con diferentes profundidades con el objetivo de comparar los resultados analíticos y el ensayo respectivo; determinar la variación entre la simulación y el modelo físico, de los cuales el error máximo fue de 3.67 \% a $15 \mathrm{~mm}$ de profundidad y el mínimo de $0.24 \%$ con la profundidad total. Con respecto a los esfuerzos generados durante el conformado, los errores máximos y mínimos fueron de $10.9 \%$ y $2.18 \%$, respectivamente.
\end{abstract}

\section{Palabras clave}

Aluminio; conformado; esfuerzo; elementos finitos; simulación.

\begin{abstract}
The research evaluates the behavior of a material when a plastic deformation occurs. The document describes the analytical process, finite elements and integrate process in order to develop shaped piece plan. Stress analysis and section variation were analyzed during the stuffing process. In the experimental part, the mechanical characteristics of the aluminum ASTM A1200 were determined (Yield strength was 214 MPa). The shaping process was carried out in a 5 tons press with pneumatic device. Several tests were executed with different depths in order to compare thicknesses with the physical model simulation. The maximum error was $3.67 \%$ at $15 \mathrm{~mm}$ depth and the minimum of $0.24 \%$ with the total depth. Respect to the efforts generated during the conforming, the maximum and minimum errors were $10.9 \%$ and $2.18 \%$, respectively.
\end{abstract}

\section{Keywords}

Aluminum; conformed; strain; finite elements; simulation.

\section{Introducción}

La industria ecuatoriana muchas veces se ha basado en métodos empiricos en los procesos de manufactura. Industrias especializadas en estampado mediante el uso de matrices utilizan materiales costosos para su fabricación, además de un considerable tiempo. Es por ello que programas de simulación computacional permiten al departamento de ingeniería predecir de manera aproximada los fenómenos físicos inherentes. Bajo este precepto se busca interrelacionar práctica con simulación, como manera viable para la ejecución de proyectos industriales. 
El término embutición viene de embutir que significa rellenar de una cierta sustancia en recipiente cerrado; delimitado por paredes flojas, para hincharlo y modificar su configuración (como los colchones). Sin embargo, en el caso de la chapa no se verifica exactamente un rellenamiento con otra sustancia; la chapa plana en origen, solamente se hincha con el fin de obtener un objeto de forma hueca, como, por ejemplo, un cubilete, una vasija, una copa, etc. Se puede emplear el término embutido para indicar la operación mediante la cual se somete a una chapa bajo la forma de un cuerpo hueco (Rossi, 1979: 66).

En el país la sinergia entre software y proceso físico es todavía relegado a empresas grandes, la finalidad de este estudio es demostrar la viabilidad y el ahorro para las Pymes al utilizar herramientas computacionales en el mejoramiento de sus procesos.

Es necesario indicar el análisis por el método de elementos finitos en la interacción de herramientas computacionales de ingeniería, ayuda a mejorar la conceptualización de especificaciones, requerimientos y trabajar con los parámetros de borde definidos y así obtener resultados óptimos para la aplicación física termo mecánica del comportamiento de los materiales (Vinueza Lozada y Gutierrez Suquillo, 2018: 202).

Permitirá al lector comprender los diferentes parámetros físicos tales como esfuerzo de fluencia, módulo de tangencia, módulo de elasticidad, que necesita un software, para simular un proceso de embutición y así predecir fallas de diseño en las herramientas en las herramientas utilizadas.

Adicionalmente, se pretende validar los algoritmos y métodos para el cálculo de fuerzas y desplazamientos recomendados por los libros. Finalmente, se busca fomentar la investigación, romper paradigmas y tecnificar los procesos de la industria.

\section{Materiales y métodos}

El proceso de embutición implica la deformación de una lámina o chapa metálica utilizando la combinación de cargas que generan esfuerzos a tensión y compresión con el objetivo de conservar el espesor de pared constante, en la Figura 1 se detalla el comportamiento de un material durante el proceso de deformación.

Figura 1. Comportamiento durante el proceso de embutido

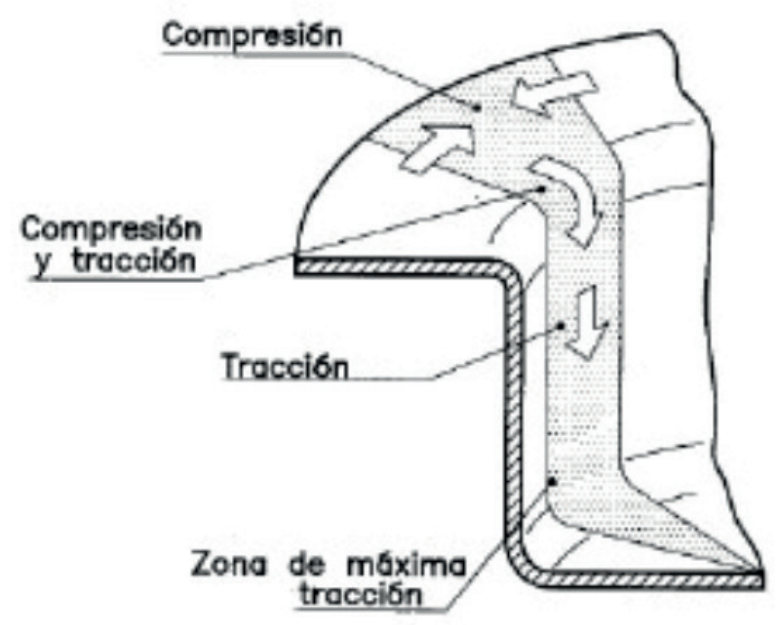


Para provocar la deformación es necesario generar cargas, el sistema que se utilizó para validar los ensayos fue una prensa con colchón neumático de 5 t de fuerza. En el proceso de embutido es necesario dos fuerzas, una que permite mantener la lámina plana y rígida denominada fuerza de sujeción y la otra que provoca la transformación plástica.

\subsection{Fuerza de embutido}

\section{Método 1}

Groover (2007), utiliza esta ecuación para la obtención de la fuerza de embutido, el valor de 0.7 es un factor de corrección para la fricción (456):

$$
F=\pi \times D_{p} \times t \times(T S) \times\left(\frac{D_{b}}{D_{p}}-0,7\right)
$$

Donde:

$F=$ Fuerza de embutido N Lbf

$t$ = Espesor original del material o la lámina $\mathrm{mm} \quad \mathrm{Plg}$

$T S=$ Resistencia a la tensión del material $\quad \mathrm{MPa} \quad \mathrm{Lbf} / \mathrm{plg}^{2}$

$D_{\mathrm{b}}=$ Diámetro del disco en forma inicial $\quad \mathrm{mm} \quad \mathrm{Plg}$

$D_{\mathrm{p}}=$ Diámetro del punzón $\quad \mathrm{mm} \quad \mathrm{Plg}$

\section{Método 2}

Kalpakjian (2008: 453) expresa la ecuación para la obtención de la fuerza:

$$
F_{\text {máx }}=\pi \times D_{p} \times T \times(U T S) \times \ln \frac{D_{o}}{D_{p}}
$$

Donde:

$\begin{array}{lll}F_{\text {máx }}=\text { Fuerza de embutido } & \mathrm{N} & \mathrm{Lbf} \\ T=\text { Espesor del material a usar } & \mathrm{mm} & \mathrm{Plg} \\ \text { UTS = Resistencia a la tensión del material } & \mathrm{MPa} & \mathrm{Lbfl}^{2}{ }^{2} \\ D_{\mathrm{o}}=\text { Diámetro del disco en forma inicial } & \mathrm{mm} & \mathrm{Plg}^{2} \\ D_{\mathrm{p}}=\text { Diámetro del punzón } & \mathrm{mm} & \mathrm{Plg}\end{array}$

\section{Método 3}

De acuerdo con Oehler y Kaiser, para la obtención de la fuerza de embutición es más fácil de obtener por métodos gráficos. (1977: 317), en la Figura 2 se describe el diagrama para establecer la fuerza del punzón, en función de la relación de embutición [A], propiedad del material [B], espesor de chapa [E], el diámetro del embutido [F] y finaliza en [G] donde se encuentra la fuerza de embutición de forma rápida y práctica. 
Figura 2. Diagrama para determinar la fuerza de embutido según Oehler y Kaiser

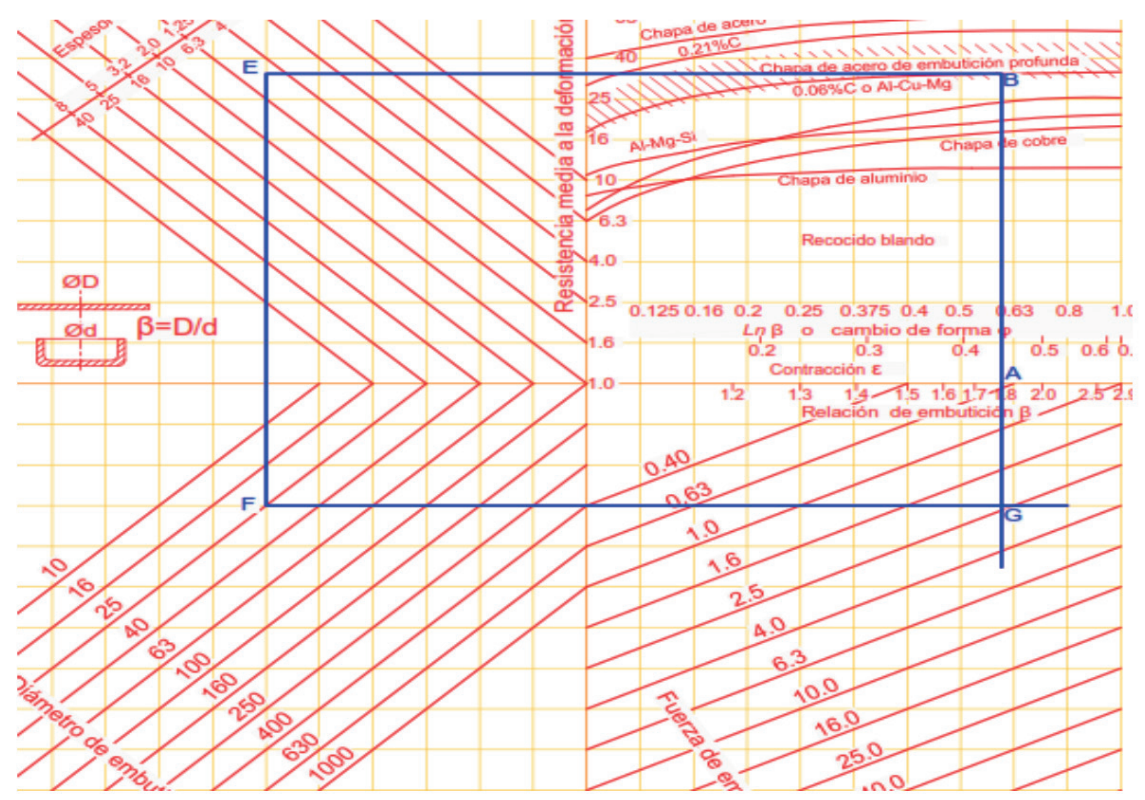

\section{Método 4}

Otro método gráfico, es determinado por López Navarro (1976: 124), como se muestra en la Figura 3, considera el diámetro del punzón, la relación d/D, el espesor de la chapa, y la resistencia del material.

Figura 3. Diagrama para determinar la fuerza de embutido según López Navarro

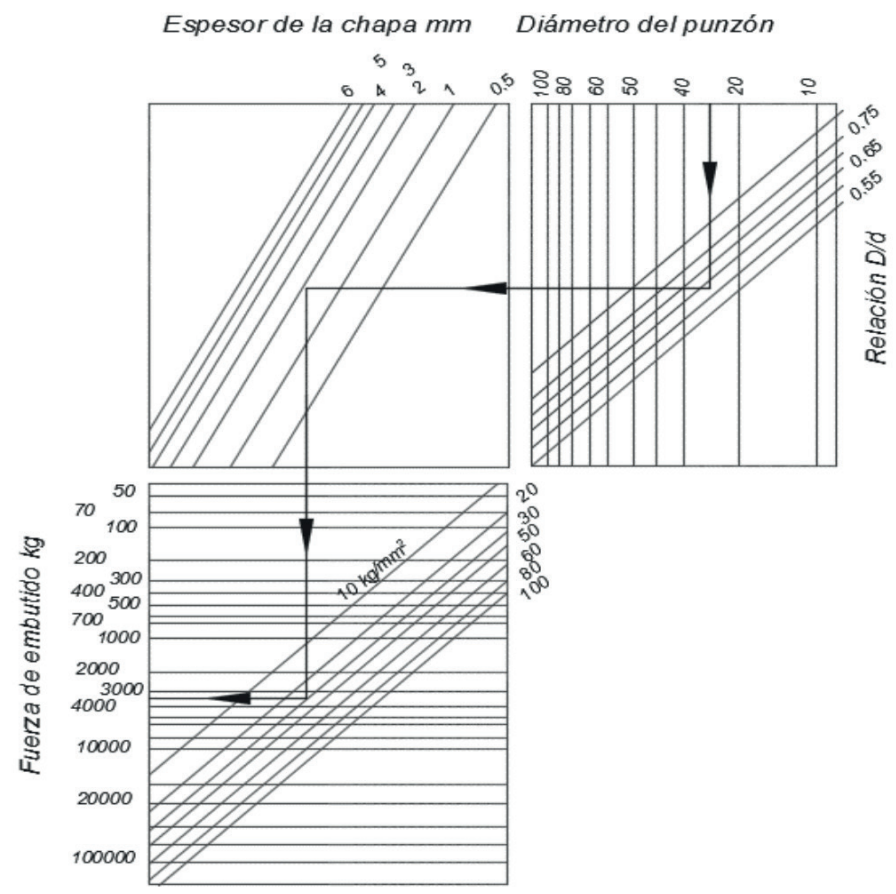




\subsection{Fuerza de sujeción}

\section{Método 1}

Groover (2007: 456), expresa esta ecuación para la obtencion de la fuerza, el valor de 0,015 es una constante de la resistencia a la fluencia de la lámina de metal:

$$
F_{h}=0,015 \times Y \times \pi \times\left[D_{b}{ }^{2}-\left(D_{P}+2.2 t+2 R_{d}\right)^{2}\right]
$$

Donde:

$F_{\mathrm{h}}=$ Fuerza de sujeción en la embutición N Lbf

$Y=$ Resistencia a la fluencia de la lámina

$t=$ Espesor original de la lámina $\mathrm{MPa}$

$R_{\mathrm{d}}=$ Radio de la esquina del troquel

$\mathrm{mm} \quad \mathrm{Plg}$

$D_{\mathrm{b}}=$ Diámetro del disco en forma inicial

$\mathrm{mm}$

Plg

$D_{\mathrm{p}}=$ Diámetro del punzón

$\mathrm{mm}$

Plg

$\mathrm{mm} \quad \mathrm{Plg}$

Método 2

De acuerdo con Rossi (1979: 104), la fuerza en el sujetador se obtiene:

$$
P=\frac{\pi}{4} \times\left(D^{2}-d^{2}\right) \times p
$$

Donde:

$\begin{array}{ll}P=\text { Fuerza total del sujetador } & \text { Kgf } \\ D=\text { Diámetro del disco en forma inicial } & \mathrm{cm} \\ d=\text { Diámetro del agujero de la matriz } & \mathrm{cm} \\ p=\text { Presión específica del sujetador } & \\ \text { Para chapa de hierro esta entre 10 a 20 } & \\ \text { Para chapa de aluminio esta entre 8 a 10 } & \mathrm{Kgf} / \mathrm{cm}^{2}\end{array}$

\section{Método 3}

López Navarro (1976: 123), determina por medio del diagrama de la Figura 4 la fuerza para el sujetador, en funcion de la diferencia de los cuadrados del diametro de la chapa desarrollada y del diametro de la pieza terminada. Se debe tener la diferencia de los cuadrados de los diámetros de la chapa desarrollada $D$ y del diámetro de la pieza terminada $d$, donde $D^{2}$ - $d^{2}$, ya conocido ese valor y la resistencia del material en $\mathrm{kg} / \mathrm{cm}^{2}$ se puede determinar la fuerza del sujetador. 
Figura 4. Diagrama para determinar la fuerza del sujetador, según López Navarro

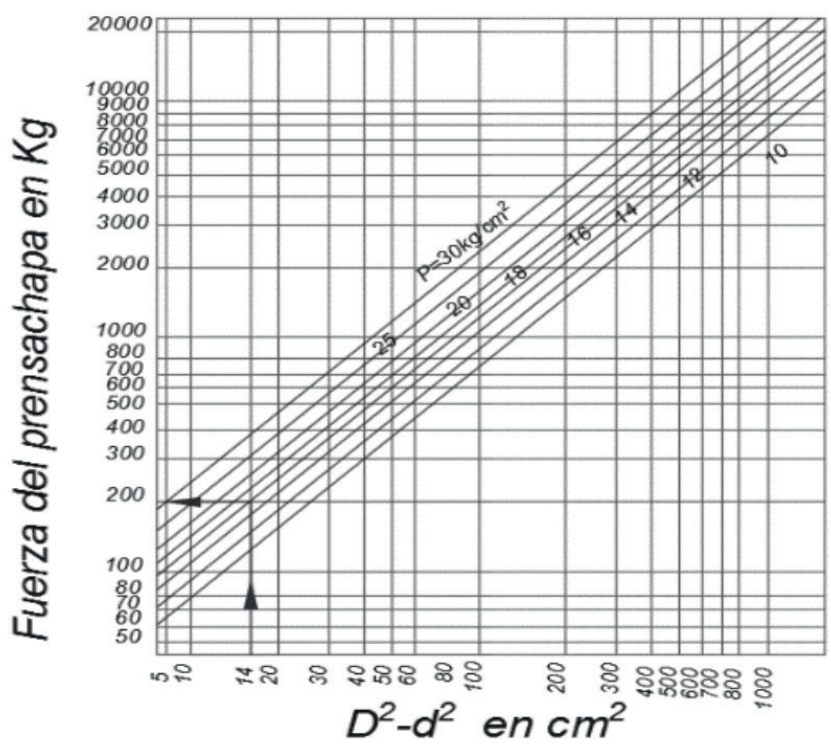

\subsection{Desarrollo de la lámina}

Previo al proceso embutido es necesario generar un elemento plana con superficie equivalente al producto final embutido, para lo cual se utiliza el método de doble integral para encontrar cada area que conforma la probeta embutida como se expresa en la Figura 5.

Figura 5. Modelo embutido
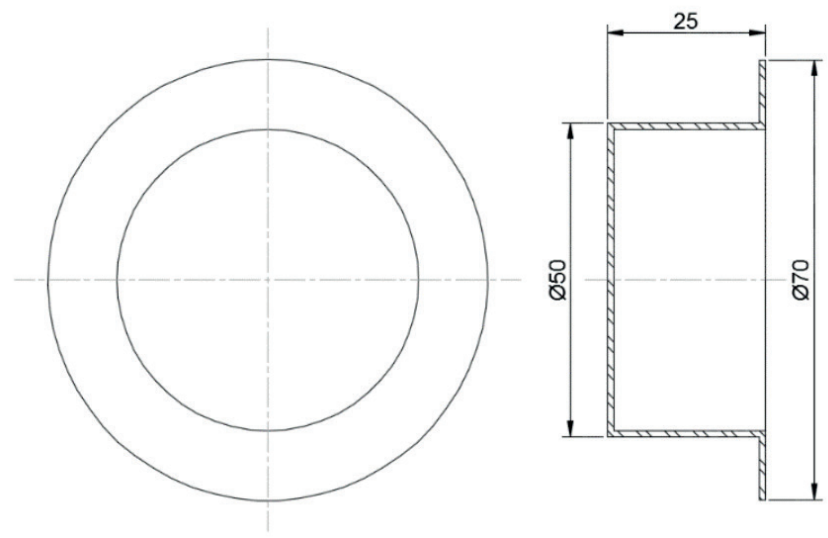

Las ecuaciones matemáticas para cada superficie se expresan a continuación.

$$
s=\int_{x 1}^{x 2} \int_{f 1(x)}^{f 2(x)} d y d x
$$




$$
\begin{array}{cc}
S_{1}=4 \int_{0}^{r} \sqrt[2]{r^{2}-x^{2}} d x & S_{1}=\pi r^{2} \\
S_{2}=4\left[\int_{0}^{R} \sqrt[2]{R^{2}-x^{2}} d x-\int_{0}^{r} \sqrt[2]{r^{2}-x^{2}} d x\right] & S_{2}=\pi\left(R^{2}-r^{2}\right) \\
S_{3}=2 \pi \int_{0}^{h} \sqrt[2]{r^{2}-y^{2}} \sqrt[2]{1+\frac{y^{2}}{r^{2}-y^{2}}} d y & S_{3}=2 \pi r h \\
S_{\text {total }}=\pi R^{2}+2 \pi r h &
\end{array}
$$

La superficie total es equivalente a la superficie del disco desarrollado, por tal motivo la expresión se establece de la siguiente manera:

$$
\begin{gathered}
\frac{\pi D^{2}}{4}=\frac{\pi d_{2}^{2}}{4}+\frac{2 \pi d_{1} h}{2} \\
D=\sqrt[2]{d_{2}^{2}+4 d_{1} h}
\end{gathered}
$$

Donde:

$D$ = Diámetro del disco desarrollado

$d_{2}=$ Diámetro mayor del modelo embutido

$d_{1}=$ Diámetro menor del modelo embutido

$h=$ Altura de embutido

\subsection{Simulación}

El proceso de simulación se realiza en un programa especializado, y su sustento matemático lo formula a través del análisis por elementos finitos, que básicamente es un método de aproximación, que divide a un sistema entero (dominio), en subsistemas (subdominios), que están unidos por nodos en sus contornos, donde se desee resultados en particular, al cual se aplica condiciones iniciales y de contorno de fuerza, tensión, deformación, temperatura.

(Zienkiewicz, 2007: 23) El continuo se divide, mediante líneas o superficies imaginarias, en un número de "elementos finitos". Se supone que los elementos están conectados entre sí por medio un número discreto de puntos, que llamaremos nodos, situados en sus contornos. Los desplazamientos de estos nodos serán las incógnitas fundamentales del problema, tal como ocurre en el análisis simple de estructuras.

\subsubsection{Fuerzas}

"El elemento a en conjunto con la carga distribuida $p$ y las fuerzas actuantes en los tres nodos 1 , 2, 3 representados en forma matricial mediante los ejes u y v, se obtiene" (Zienkiewicz, 2007: 6). 


$$
\mathbf{q}^{1}=\left\{\begin{array}{l}
\mathbf{q}_{1}^{1} \\
\mathbf{q}_{2}^{1} \\
\mathbf{q}_{3}^{1}
\end{array}\right\} ; \mathbf{q}_{1}^{1}=\left\{\begin{array}{l}
\mathrm{U}_{1} \\
\mathrm{~V}_{1}
\end{array}\right\}, \text { etc. }
$$

Donde:

$\mathbf{q}^{1}=$ Matriz columna de fuerzas en todos los nodos del elemento a.

$\mathbf{q}_{1}^{1}, \mathbf{q}_{2}^{1}, \mathbf{q}_{3}^{1}=$ Matriz de fuerzas en cada uno de los nodos 1, 2, 3 .

$\mathrm{U}_{1}, \mathrm{~V}_{1}=$ Componentes de fuerza en cada uno de los nodos en los ejes $u \mathrm{y} v$.

\subsubsection{Desplazamientos}

Según (Zienkiewicz, 2007: 6) los desplazamientos nodales se expresan de la siguiente forma:

$$
\mathbf{a}^{1}=\left\{\begin{array}{l}
\mathbf{a}_{1}^{1} \\
\mathbf{a}_{2}^{1} \\
\mathbf{a}_{3}^{1}
\end{array}\right\} ; \mathbf{a}_{1}^{1}=\left\{\begin{array}{l}
\mathrm{u}_{1} \\
\mathrm{v}_{1}
\end{array}\right\}, \text { etc. }
$$

Ec. 13

\section{Donde:}

$\mathbf{a}^{1}=$ Matriz de desplazamiento del elemento a.

$\mathbf{a}_{1}^{1}, \mathbf{a}_{2}^{1}, \mathbf{a}_{3}^{1}=$ Matriz de desplazamiento en cada uno de los nodos 1, 2, 3 .

$\mathrm{U}_{1}, \mathrm{~V}_{1}=$ Componentes de desplazamiento en cada nodo, en los ejes $u$ y $\mathrm{v}$.

\subsubsection{Tensiones}

Relacionando las fuerzas con los desplazamientos y suponiendo un comportamiento elástico del sistema la relación característica se expresa de la siguiente manera:

$$
\mathbf{q}^{1}=\mathbf{K}^{1} \mathbf{a}^{1}+\mathbf{f}_{\mathrm{P}}^{1}+\mathbf{f}_{\varepsilon \mathbf{o}}^{1}
$$

Donde:

$\mathbf{q}^{1}=$ Matriz de fuerzas en todos los nodos del elemento a.

$\mathbf{K}^{1}$ = Matriz de rigidez del elemento a

$\mathbf{a}^{1}=$ Matriz desplazamiento del elemento a.

$\mathbf{f}_{\mathrm{P}}^{1}=$ Matriz de fuerzas nodales necesarias para equilibrar cualquier carga distribuida que actúe sobre el elemento

$\mathbf{f}_{\varepsilon \mathrm{o}}^{1}=$ Matriz de fuerzas nodales necesarias para equilibrar cualquier deformación inicial. 


\section{Resultados}

\subsection{Ensayo de probetas}

Se realizó ensayos de tracción en material de aluminio ASTM A1200 con un espesor 0,5 mm en los laboratorios de análisis de esfuerzos y vibraciones (LAEV) de la Escuela Politécnica Nacional, las dimensiones de la probeta para los ensayos se desarrolló según la norma ASTM E8. En la Figura 6 se indica la probeta antes y luego del ensayo realizado.

Figura 6. Ensayo de probetas

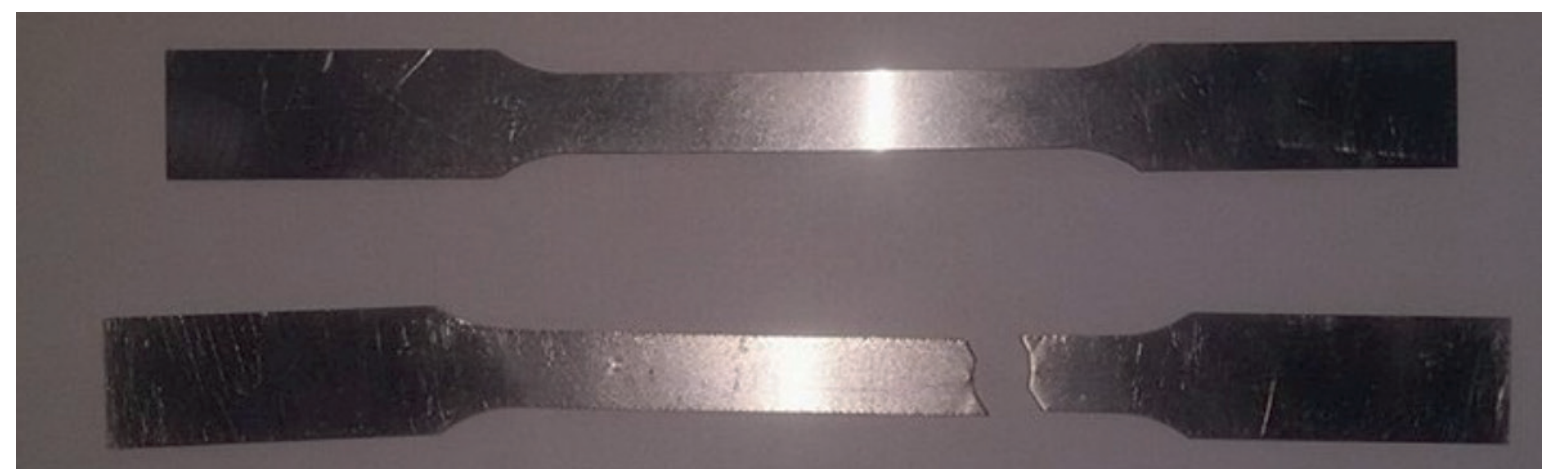

Los ensayos presentaron un esfuerzo de fluencia de 214 MPa y el esfuerzo último del material 275 MPa y la curva de ensayo se indica en la Figura 7.

Figura 7. Curva esfuerzo deformación

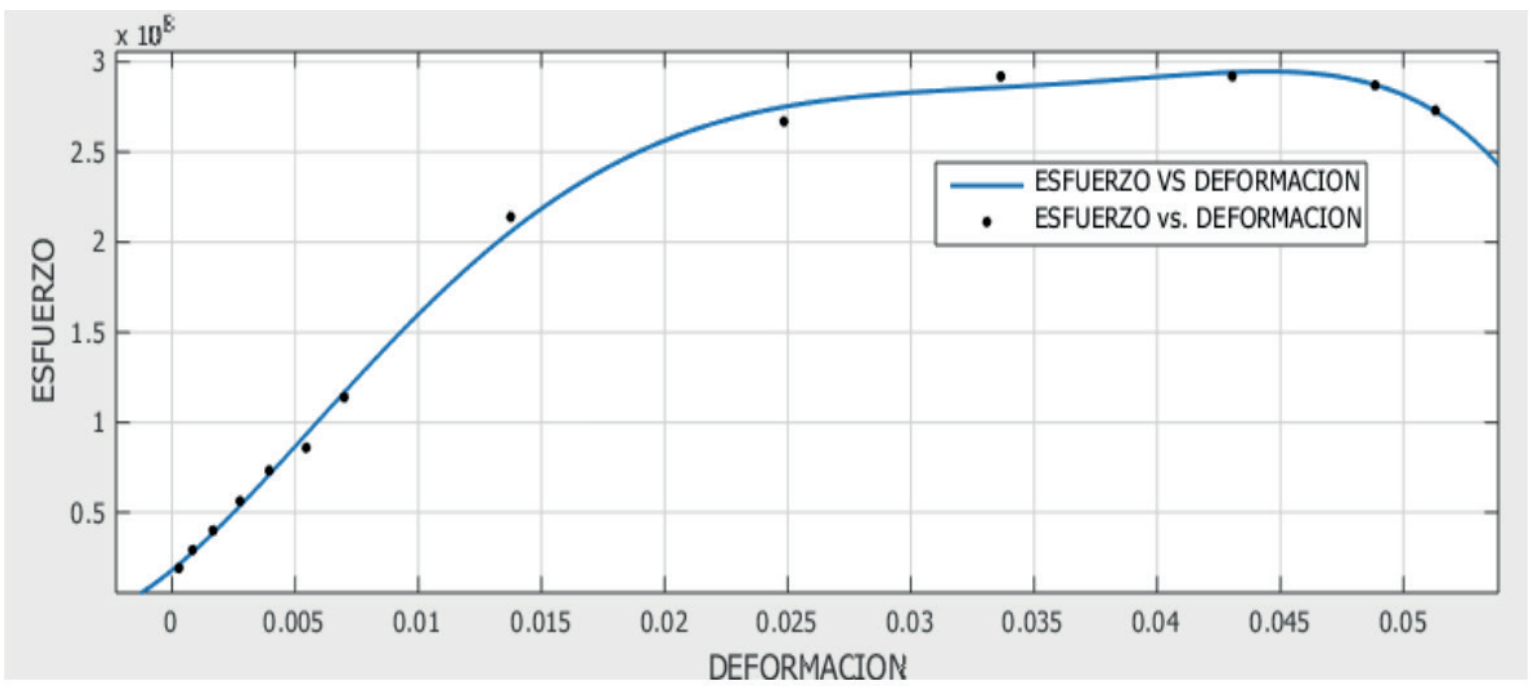

\subsection{Determinación del desarrollo}

Una vez desarrollado la doble integral, el valor del diámetro mínimo requerido para el embutido a estudiar es de 99.5 mm, en la Figura 8 se detalla la generación del modelo en plano mediante software. 
Figura 8. Desarrollo de la pieza en plano
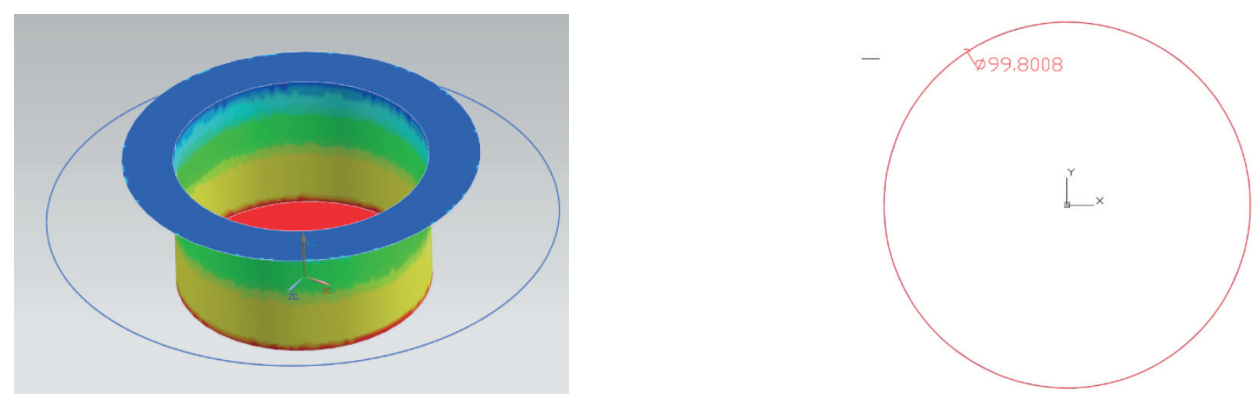

\subsection{Fuerza de embutido}

En la Tabla 1 se describe los valores utilizados para la obtención de la fuerza teórica necesaria.

Tabla 1. Datos utilizados para el cálculo de la fuerza de embutido

\begin{tabular}{|l|c|c|c|c|c|c|}
\hline & Método 1 & Método 2 & Método 3 & Método 4 & Valor & Unidades \\
\hline Espesor & $\mathrm{t}$ & $\mathrm{T}$ & $\mathrm{t}$ & $\mathrm{t}$ & 0.50 & $\mathrm{~mm}$ \\
\hline Diámetro de forma inicial & $\mathrm{D}_{\mathrm{b}}$ & $\mathrm{D}_{0}$ & $\mathrm{D}$ & $\mathrm{D}$ & 99.5 & $\mathrm{~mm}$ \\
\hline Diámetro del punzón & $\mathrm{D}_{\mathrm{b}}$ & $\mathrm{D}_{\mathrm{b}}$ & - & $\mathrm{d}$ & 48.7 & $\mathrm{~mm}$ \\
\hline Diámetro del embutición & - & - & $\mathrm{d}$ & - & 50.0 & $\mathrm{~mm}$ \\
\hline Resistencia a la tensión & TS & UTS & - & - & 251.09 & $\mathrm{MPa}$ \\
\hline
\end{tabular}

En la Tabla 2 se detallan las fuerzas calculadas y la fuerza real aplicada durante el ensayo.

Tabla 2. Fuerza utilizada para el embutido

\begin{tabular}{|l|l|l|l|}
\hline & \multicolumn{1}{|c|}{ Fuerza calculada } & \multicolumn{1}{|c|}{$\begin{array}{c}\text { Fuerza real (presión } \\
\text { del equipo 1200 PSI) }\end{array}$} & \multicolumn{1}{|c|}{ Error } \\
\hline Método 1 & $2632,49 \mathrm{Kgf}$ & $2285 \mathrm{Kgf}$ & $13.2 \%$ \\
\hline Método 2 & $1436,41 \mathrm{Kgf}$ & $2285 \mathrm{Kgf}$ & $37.1 \%$ \\
\hline Método 3 & $1020,00 \mathrm{Kgf}$ & $2285 \mathrm{Kgf}$ & $55.4 \%$ \\
\hline Método 4 & $2500,00 \mathrm{Kgf}$ & $2285 \mathrm{Kgf}$ & $8.6 \%$ \\
\hline
\end{tabular}

\subsection{Fuerza del presachapa}

En la Tabla 3 se detalla los parámetros utilizados para el cálculo de la fuerza de sujeción para el proceso de embutido.

Tabla 3. Datos utilizados para el cálculo de la fuerza de sujeción

\begin{tabular}{|l|c|c|c|c|c|c|}
\hline & Método 1 & Método 2 & Método 3 & Método 4 & Valor & Unidades \\
\hline Espesor & $\mathrm{t}$ & $\mathrm{t}$ & $\mathrm{T}$ & $\mathrm{t}$ & 0.50 & $\mathrm{~mm}$ \\
\hline Diámetro de forma inicial & $\mathrm{D}_{\mathrm{b}}$ & $\mathrm{D}$ & $\mathrm{D}$ & $\mathrm{D}$ & 99.5 & $\mathrm{~mm}$ \\
\hline Diámetro del punzón & $\mathrm{D}_{\mathrm{b}}$ & - & - & - & 48.7 & $\mathrm{~mm}$ \\
\hline Diámetro del embutición & - & $\mathrm{d}$ & $\mathrm{d}$ & $\mathrm{d}$ & 50.0 & $\mathrm{~mm}$ \\
\hline Resistencia a la fluencia & $\mathrm{Y}$ & - & - & - & 105 & $\mathrm{MPa}$ \\
\hline Radio de esquina del punzón & $\mathrm{R}_{\mathrm{d}}$ & - & - & $\mathrm{r}$ & 2 & $\mathrm{~mm}$ \\
\hline
\end{tabular}


En la Tabla 4 se detallan los valores comparativos entre la fuerza teórica y la fuerza real aplicada durante la parte experimental.

Tabla 4. Fuerza utilizada para en la sujeción

\begin{tabular}{|l|c|c|c|}
\hline & Fuerza calculada & $\begin{array}{l}\text { Fuerza real (presión } \\
\text { del equipo 250 PSI) }\end{array}$ & Error \\
\hline Método 1 & $884.31 \mathrm{Kgf}$ & $702 \mathrm{Kgf}$ & $20.6 \%$ \\
\hline Método 2 & $581.21 \mathrm{Kgf}$ & $702 \mathrm{Kgf}$ & $17.2 \%$ \\
\hline Método 3 & $550.00 \mathrm{Kgf}$ & $702 \mathrm{Kgf}$ & $21.7 \%$ \\
\hline
\end{tabular}

\subsection{Análisis de espesores}

En la Figura 9 se describe los espesores logrados durante el proceso de simulación y los desarrollados en la parte experimental, en tres estados diferentes de profundidad.

Figura 9. Análisis de espesores durante el proceso de embutido

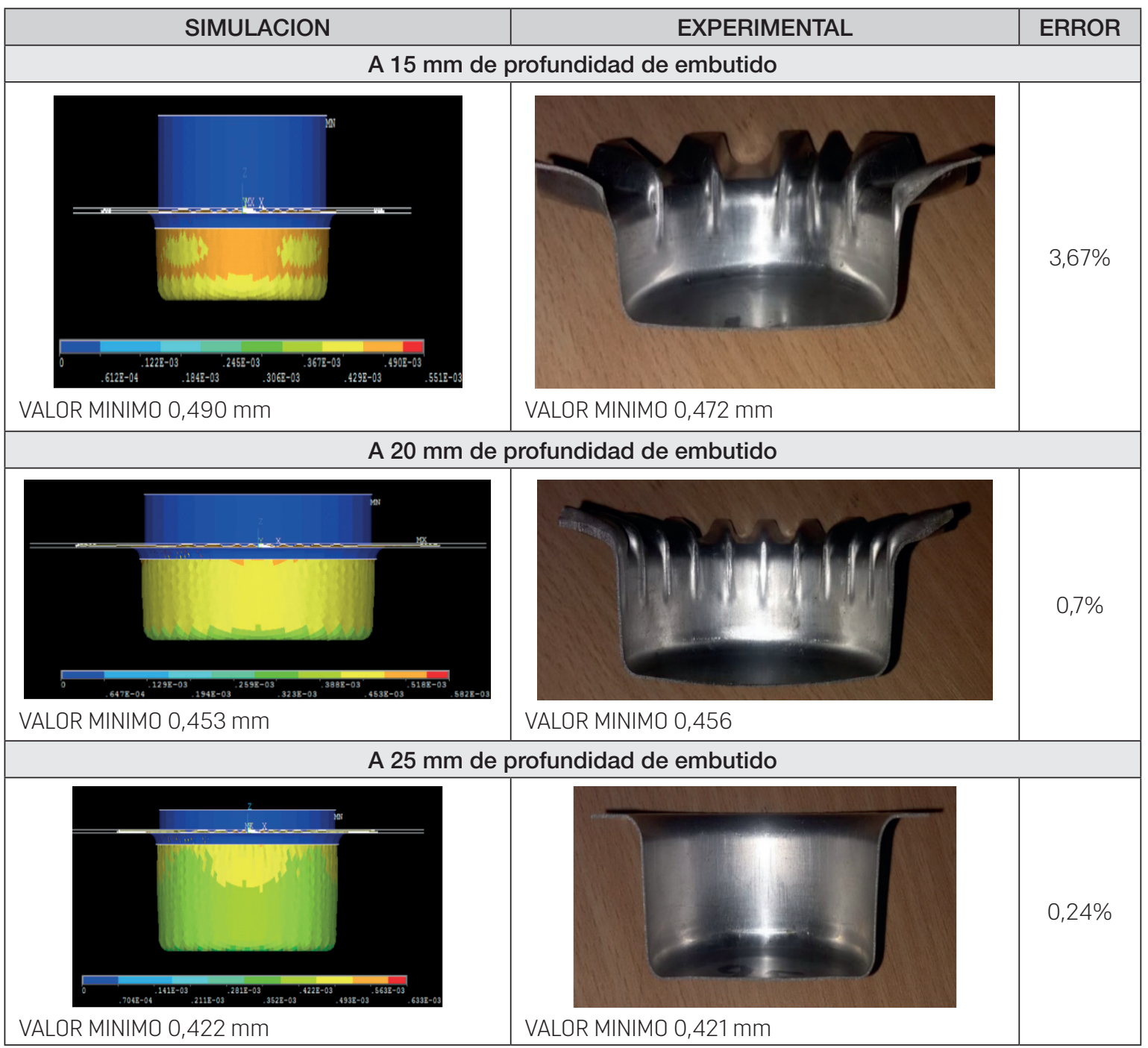




\subsection{Análisis de esfuerzos}

Analizar el esfuerzo generado durante el proceso de embutido en la simulación hecha, sirve como guía para establecer si el material puede o no ser conformado, en la Figura 10 se observan los esfuerzos provocados.

Figura 10. Análisis de esfuerzos durante el proceso de embutido

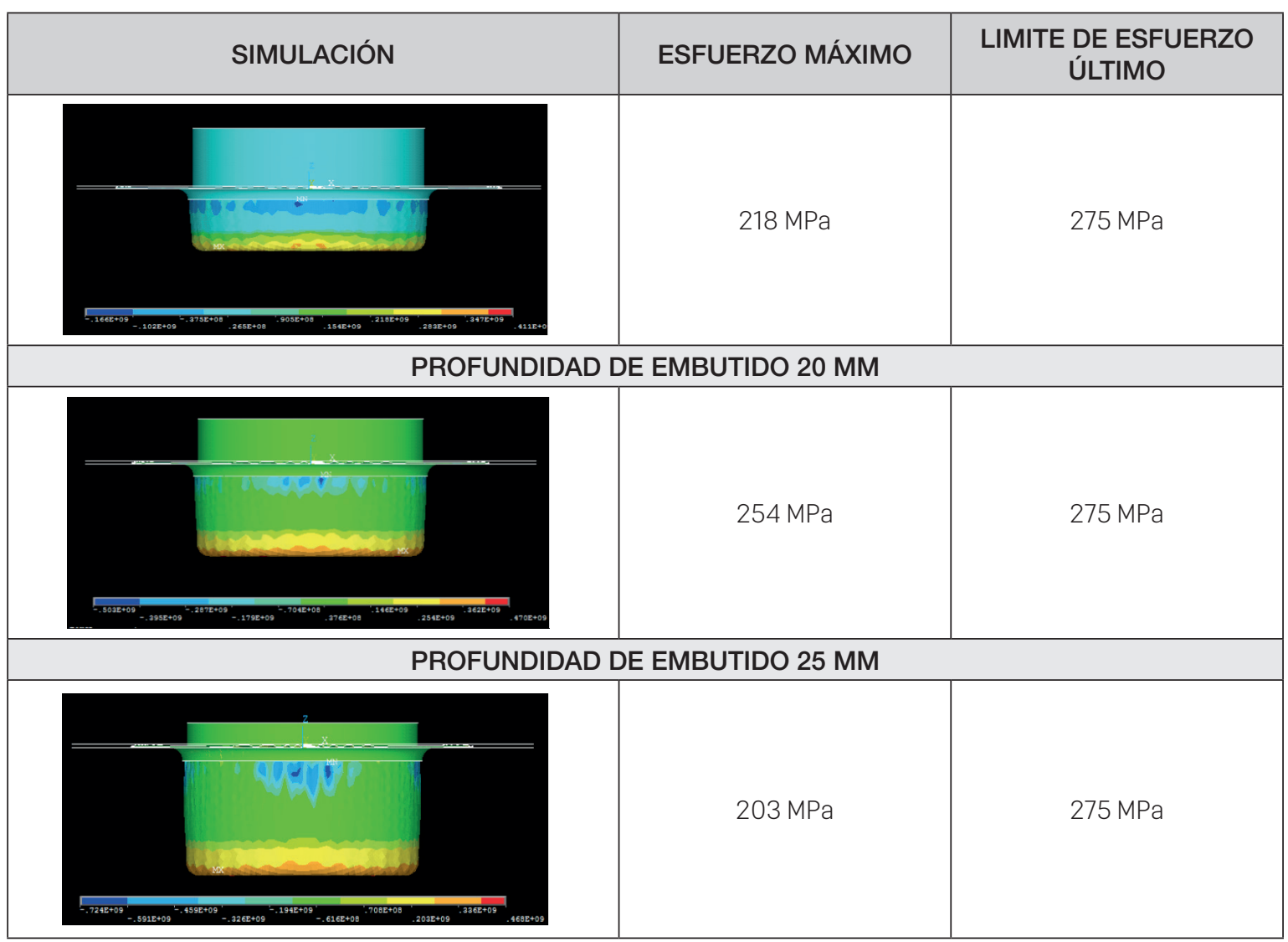

\section{Conclusiones y recomendaciones}

Para validar la simulación y la parte experimental es necesario trabajar con datos reales, por tal motivo el ensayo a tracción del material permitió utilizar un esfuerzo límite real de 275 MPa el cual fue utilizado para los cálculos analíticos y como parámetro en la simulación.

Con la presente investigación se establece que para un proceso de embutido los métodos expuestos por los autores Groover y López Navarro tienen un error de 13.8 \% y 8.2 \%, respectivamente, y sirven como guía para la configuración inicial de embutido en la parte práctica industrial.

La fuerza de sujeción tiene una vital importancia para la calidad de la probeta, con una fuerza superior a $702 \mathrm{Kgf}$, el material se fractura y no permite terminar el proceso de embutido, y por otro lado una carga inferior provoca arrugas en el producto final, por tal motivo el método dos para estimar la fuerza del pisador es el que menor error presenta.

Para un proceso de embutido a nivel industrial, la figura plana es muy importante, pues garantizará que la pieza sea conformada en su totalidad, con el presente estudio se valida la 
utilización del software para optimizar tiempos de ejecución en proyectos, con la siguiente justificación, el modelo matemático presentó un diámetro de 99.5 mm y la figura desarrollada en el software fue de $99.8 \mathrm{~mm}$, error presentado fue de 0,3\%.

Durante la simulación se presentó el máximo esfuerzo en el material antes de $20 \mathrm{~mm}$ de profundidad, el valor fue de $254 \mathrm{MPa}$, cercano al límite último, por tal motivo la regulación de velocidad disminuyó el riesgo de fractura del material.

Los límites de frontera para la simulación garantizan resultados confiables, se puede ratificar con la medición de espesores, que presenta un error máximo de 4\%, brindando un grado de confiabilidad alto para proyectos a nivel industrial.

El presente proyecto es una guía para realizar validaciones de simulación vs. ensayos experimentales con diferentes materiales, enfocado al proceso de conformado mecánico.

Para un proceso de simulación, es necesario contar con equipo computacional elevado, pues el manejo de miles de nodos y sus respectivos ralentizan equipos convencionales de computación.

\section{Referencias}

ANSYS. (2013). ANSYS Mechanical APDL Basic Analysis Guide. Pensilvania: SAS IP.

Bernal Aguilar, Y. (2013). Planeación de procesos de embutido de chapa mediante herramientas numéricas. Revista Centro Azúcar, 29-38.

Bravo Vargas, S., y Reta, C. (2016). Desarrollo de un modelo virtual para conformado de aceros inoxidables. 8va. Congreso internacional de Ingeniería Electromecánica y de Sistemas, 1-6.

Campos Vasquéz, A., Escamilla Navarro, A., y González López, A. (2013). Análisis experimental-numérico de la plantilla para el conformado plástico mediante embutido de una abrazadera metálica. Cientifica, 67-76.

Escribano, A. I., y Herranz, R. A. (2014). DEDUCETU. Retrieved from DEDUCETU: http://de-duce-tu.blogspot. com/p/embuticion-2.html

Fuentes Quisaguano, M. X., y Mullapa Rumipamba, M. (2008, Enero). Matriz de embutición y corte para la implementación del laboratorio de matricería. Quito. Retrieved from Repositorio Digital EPN: http://bibdigital.epn.edu.ec/bitstream/15000/2005/1/CD-1202.pdf

Groover, M. P. (2007). Fundamentos de Manufactura Moderna (3ª. ed.). CDMX, México: The McGraw-Hill.

Kalpakjian, S. (2008). Manufactura, ingeniería y tecnología (5a. ed.). CDMX, México: Pearson Educación.

Kurt, M., y Juvinall, C. R. (2013). Diseño de elementos de maquinas. madrid: Limusa.

López Navarro, T. (1976). Troquelado y Estampación (5a. ed.). Barcelona, España: Gustavo Gili.

Oehler, G., y Kaiser. (1977). Herramientas de troquelar, estampar y embutir (6ª ed.). Barcelona, España: Gustavo Gili.

Osrodala. (junio 9 de 2012). Wikemedia Commons. Retrieved from Wikemedia Commons: https://commons.wikimedia.org/wiki/File:EMBUTIDO_general.jpg

Rodríguez, M., y González, A. (2012). Elasticidad y resistencia de materiales I. Madrid, España: Universidad Nacional de Educación a Distancia.

Rossi, M. (1979). Estampado en frío de la chapa (9a. ed.). Madrid, España: DOSSAT, S.A.

Villamar Palacios, V. H. (2008). Diseño de una prensa hidraulica para elaborar pocetas de acero inoxidable. Guayaquil: s. e.

Vinueza Lozada, A. F., y Gutierrez Suquillo, N. R. (2018). Análisis por el método de elementos finitos del comportamiento de las pastillas de freno ABS con base de acero y zinc al discretizar el elemento contínuo al utilizar software CAE. Enfoque UTE, 188-203.

Zienkiewicz, O. C. (2007). El método de los elementos finitos. Barcelona, España: Reverté. 